\title{
Broad-band Active Vibration Suppression using PPF focused on Industrial Application*
}

\author{
A.J. den Hamer ${ }^{1)}$, G.Z. Angelis ${ }^{2)}$, N.B. Roozen ${ }^{2,3)}$ \\ 1) Control Systems Technology Group, Department of Mechanical Engineering, \\ Eindhoven University of Technology, 5600 MB Eindhoven, The Netherlands. \\ 2) PHILIPS Centre for Industrial Technology, 5600 MD Eindhoven, The Netherlands. \\ 3) Dynamics and Control Group of the Department of Mechanical Engineering, \\ Eindhoven University of Technology, 5600 MB Eindhoven, The Netherlands.
}

\section{Summary}

Due to the demand for higher accuracy and lower acoustic noise levels, there is a growing interest for active vibration suppression in industrial application. Many control concepts have been proposed in this field that are suited to introduce damping in systems with a large number of modes. However tuning is often focussed on reduction of one dominant mode. Such control approaches are less transparent when several dominant modes are involved.

A combined loop-shaping and root-locus tuning approach is proposed to reduce several dominant modes with low-order controllers. The tuning approach is experimentally evaluated on a vibrating plate using piezo's for both sensing and actuating. Based on the given tuning rules and trade-offs, a SISO feed-back controller is designed and implemented. This low authority control approach shows that besides local performance, also the global damping of the plate is improved which consequently results in reduction of vibration levels over the whole plate for a broad range of frequencies. As a results, acoustic noise radiation is reduced significantly.

\section{1 introduction}

Vibration problems typically occur in badly damped distributed mechanical systems. Their high modal density and badly damped modes can lead to a broad spectrum of vibrations of relative high amplitude [1]. This leads at the one hand to noise radiation via mechanical-acoustic coupling, on the other hand to loss of position accuracy. To reduce these effects, active vibration suppression has gained interest above passive damping due to better tunable suppression especially at lower frequencies and a lower amount of added mass.

The high modal density of the structure and the

*Preprint submitted to IEEE/ASME TRANSACTIONS ON MECHATRONICS, 2005 lack of roll-off adds additional constraints on the type of controller. In literature, several control approaches are proposed in the field of active vibration suppression $[2,3,4,5,6,7]$. These approaches can be roughly split into high authority and low authority controllers.

The performance of high authority controllers such as $\mathcal{H}_{2}$ and $\mathcal{H}_{\infty}$ appear to be very sensitive to model uncertainty and modelling errors as introduced by model truncation $[6,8]$. On the other hand, using accurate high order models for synthesis of these controllers leads to practical limitations. The resulting high order controllers need to be reduced in order to enable implementation in state-of-the-art digital controllers. The order truncation however, causes a mismatch which reduces control performance or even results in an unstable closed-loop system.

Low authority methods such as velocity feedback (VF) and positive position feedback (PPF) are proposed which can overcome the disadvantages mentioned above $[4,5]$. This paper will be focussed on the tuning of low authority controllers using collocated sensor and actuator pairs.

Tuning of low authority controllers is often focussed on damping of a single dominant mode [7, 2, 9]. This does not comply with the industrial demands which often ask for a more broad-banded approach [10]. This paper will show that adding damping in a broad spectrum of resonances is possible using a controller with low complexity whereas the tuning is based on both root-locus and the Frequency Response Function (FRF) of the open-loop.

The approach is evaluated for a flexible plate, which is an example of a distributed system, often used in industry as a light and stiff construction element.

\section{2 experimental setup}

The experimental setup as depicted in Fig. 1(a) consists of a flexible aluminium plate mounted in a rigid box as designed by Twente University [11]. Information about geometry can be found in Table 2 whereas 
the dimensions are given with respect to the coordinate frame in Fig. 1(b). Information about mode shapes and resonance frequencies can be found in Table 3 .

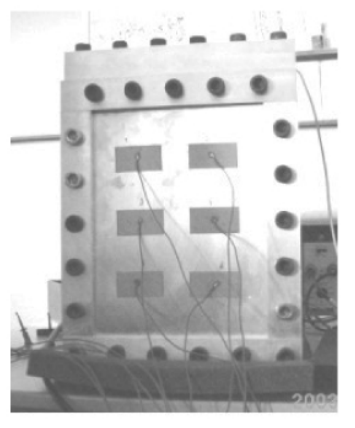

(a)

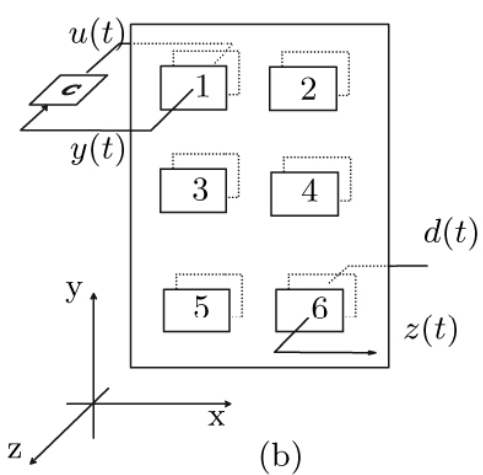

(b)
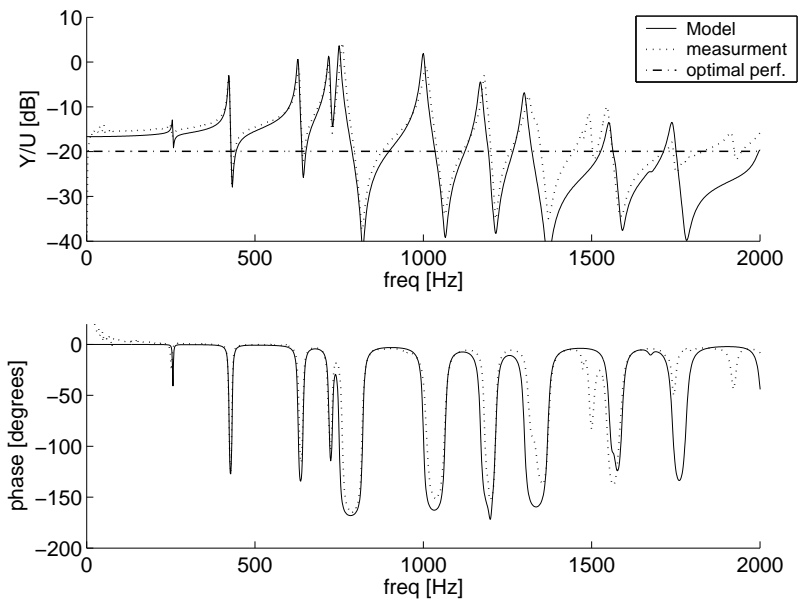

Figure 2: Frequency Response Function $\frac{Y(j \omega)}{U(j \omega)}$

Figure 1: a) Experimental setup, b) Schematic configuration

Table 1: Dimension of experimental setup

\begin{tabular}{|c|c|c|c|}
\hline \multicolumn{2}{|c|}{ Plate } & \multicolumn{2}{c|}{ Piezo (each) } \\
\hline$\Delta x$ & $16 \cdot 10^{-2}[\mathrm{~m}]$ & $\Delta x$ & $4.5 \cdot 10^{-2}[\mathrm{~m}]$ \\
$\Delta y$ & $21 \cdot 10^{-2}[\mathrm{~m}]$ & $\Delta y$ & $2.3 \cdot 10^{-2}[\mathrm{~m}]$ \\
$\Delta z$ & $1.0 \cdot 10^{-3}[\mathrm{~m}]$ & $\Delta z$ & $3.0 \cdot 10^{-4}[\mathrm{~m}]$ \\
material & $\mathrm{Al}$ & material & $\mathrm{PZT}$ \\
\hline
\end{tabular}

An array of PZT-piezo's is mounted on the front and back side of the plate by means of electrically conductive glue. The piezo's at the front side are used for sensing while the piezo's at the back side serve as actuators.

The piezo pairs are numbered according to Fig. 1(b). The control loop uses the signals $u(t)$ and $y(t)$ of the piezo pair 1 whereas the level of vibration suppression is evaluated using the signals $d(t)$ and $z(t)$ of piezo pair 6 . Note that due to symmetry, the uncontrolled dynamic behaviour of $u(t)$ to $g(t)$ equals $d(t)$ to $z(t)$.

Identical locations are used for both sensing and actuating. This leads to collocated sensor-actuator pairs which proves predictable phase behaviour, bounded between 0 and $-180^{\circ}$ (Fig. 2), corresponding with an alternating pattern in the pole-zero map [2] (Fig. 6 and 7). This collocated behaviour is desirable from a stability point of view.

\section{3 modelling for performance}

The tuning of the controller, as will be described in section 5 , consist of two parts concerning a data-based part to tune for stability and model based part to tune for performance, i.e. reduction of vibration levels in a global way. The model to describe $\frac{Y(s)}{U(s)}$, needed to tune for performance, will be obtained in this paragraph.

The transfer function of a lightly damped mechanical distributed parameter system $P(s)$ can in general be written as a sum of an infinite number of weighted modal components [12],

$$
\begin{aligned}
Y(s) & =P(s) U(s) \\
P_{i j}(s) & =\sum_{n=1}^{n=\infty} \phi_{i_{n}} \phi_{j_{n}} \frac{1}{s^{2}+2 \xi_{n} \omega_{n}+\omega_{n}^{2}}
\end{aligned}
$$

where $U(s)$ and $Y(s)$ are respectively the Laplace transforms of the plant in- and outputs, i.e. the measured voltage $y(t)$ and applied voltages at the piezo $u(t)$ as depicted in Fig. 1(b). $\omega_{n}, \xi_{n} \in \mathbb{R}$ and $s \in \mathbb{C}$ are respectively the modal eigenfrequency, the modal damping and the Laplace variable. $\phi_{i}$ and $\phi_{j}$ represent the modal participation factors of input $i$ and output $j$.

Since the aim is to increase damping up to $\pm 2 \mathrm{kHz}$, only a limited number of resonances needs to be considered, allowing reduction of the model-order. For the experimental setup, the first 12 non-Rigid Body Modes (RBM) describe the given dynamics up to 2 $\mathrm{kHz}$ sufficiently accurate. The flexible behaviour, represented by Eq.(1), can in the low frequency range be approximated by a truncated serie of non-RBM:

$$
P_{i j}(s) \approx \sum_{n=1}^{n=12} \phi_{i_{n}} \phi_{j_{n}} \frac{1}{s^{2}+2 \xi_{n} \omega_{n}+\omega_{n}^{2}}+D_{i j}
$$

where the residual term $D_{i j}$ equals the steady state behaviour of the modes 13 till $\infty$ with respect to input $i$ and output $j$.

It can be observed from Eq.(1) that the modal eigenfrequency $\omega_{n}$ and modal damping $\xi_{n}$ are independent from the chosen input $i$ and output $j$. So if 
the poles are shifted to other locations, every transfer of the system is influenced according to Eq.(1). Increasing the modal damping locally will show a reduction in the corresponding modal peaks of $P_{i j}$ in a global manner.

In order to be able to shift the poles of a certain mode, these poles need to be both observable and controllable [13]. Therefore sensor and actuator locations have to be chosen such that the value of $\phi_{i_{n}}$ and $\phi_{j_{n}}$ of a targeted mode $n$ is as large as possible. Optimal locations are typically associated with area's of maximum modal strain [2] as can be observed from Eq.(3) [14] which supplies a relation between geometric mode-shapes and modal piezo-output voltages.

$\phi_{i_{n}} \approx \frac{1}{C_{r}} \int_{\Omega_{i}} z_{m}\left(\frac{e_{31} \partial^{2} w_{i_{n}}(x, y)}{\partial x^{2}}+\frac{e_{32} \partial^{2} w_{i_{n}}(x, y)}{\partial y^{2}}\right) d S$

where $w_{i_{n}}, \Omega_{i}$ and $z_{m}$ are respectively the displacement in z-direction of piezo $i$ for mode $n$ at location $(x, y)$, the surface of piezo $i$ and the distance between the midplane of the piezo and the plate. $e_{31}$ and $e_{32}$ are the piezo constants which are equal for isotropic material. $C_{r}$ denotes the capacity of the charge amplifier.

Eq.(3) can be used to predict/model the input to output behaviour of the piezo's as described in [14]. This modelling can be done by a coupled piezo/mechanical Finite Element Modelling (FEM) approach. In industrial applications however, involving complex geometries, a measurement based approach is often preferred above a model based one since it excludes model errors due to imperfect modelling and omits time consuming FEM modelling.

As a data-based approach, Laser vibrometer measurements are used to measure operational deflection shapes (ODS) and fit the modal strain shapes. Under the assumption that structural properties of the piezo are negligible, these modal strain shapes can be used to predict and model dynamic behaviour from the piezo actuators to the piezo sensors before actual placement [14].

When the piezo's are attached to the structure, the model can be improved by fine-tuning the poles, zeros and static gain based on the measured FRF. Fig. 2 depicts both the measured and modelled FRF of $\frac{Y(s)}{U(s)}$.

\section{Positive Position Feedback}

In order to introduce modal damping, several approaches have been proposed in literature. Two well known concepts are VF and PPF [4, 5]. The most important advantages and disadvantages of PPF over VF are shortly listed:

+ The infinite number of modal components of mechanical distributed systems (Eq.(1)) makes that attention has to be paid to the effect of high frequent dynamics on controller performance and stability. For this reason, the low-pass behaviour of PPF is preferred above the high-pass behaviour of $\mathrm{VF}$ in order to avoid high frequent spill-over effects.

+ PPF is easy to tune for stability because the controller gain is restricted by the static behaviour of the plant [5]. This will be shown in section 5.1.

$\pm \mathrm{PPF}$ has more tuning freedom than VF. This makes the tuning harder but can have benefits if tuning is well understood.

- A PPF controller reduces the low frequent stiffness of the controlled system $[15,16]$ which will be shortly commented in section 5.1.2.

Because of these arguments and positive findings in literature $[15,17], \mathrm{PPF}$ is chosen for implementation.

Like the system itself, a PPF controller, denoted by $C(s)$ can be written as a sum of $k$ modal components each having their own eigenfrequency and damping. Using negative feedback (see Fig. 3), the transfer function of a PPF controller can be written as

$$
\begin{aligned}
& U(s)=C(s) Y(s) \\
& C(s)=\sum_{n=1}^{n=k} G_{n} \frac{-1}{s^{2}+2 \xi_{c_{n}} \omega_{c_{n}} s+\omega_{c_{n}}^{2}}
\end{aligned}
$$

where $U(s)$ and $Y(s)$ are the control-output and input according to Fig. 1 and 3, whereas $G_{n}, \omega_{c_{n}}$ and $\xi_{c_{n}}$ are the chosen gain, eigenfrequency and damping of controller mode $n$, respectively.

The idea of PPF control is to create auxiliary Degree's of Freedom (DoFs) [18], i.e. controller modes, which join particular modal movements of the system. Due to the damping introduced by this auxiliary DoF, energy is dissipated from the modes in which the additional DoFs participate. This can be physically interpreted using the analogy of a tuned-mass-damper vibration absorber as stated in [16].

\section{Controller tuning}

A combined approach of both root-locus and openloop tuning is proposed. Open-loop information is used to tune for stability and to derive limits of performance whereas root-locus is used to tune for performance by shifting initially undamped poles of the system into the left half-plane, as shortly mentioned in section 3. Afterwards both methods are linked together.

\subsection{Open-loop}

\subsubsection{Local vs. global performance}

Closing the control-loop as depicted in Fig. 3 creates new dynamic behaviour of the plant which is denoted 


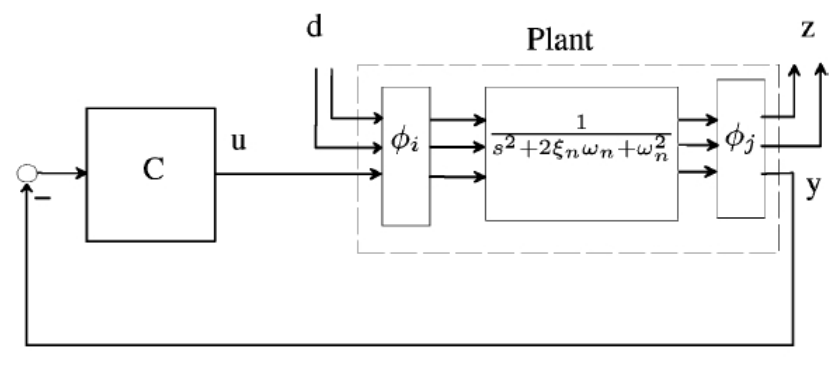

Figure 3: SISO closed-loop configuration

as $P_{c l}$. The local SISO closed-loop disturbance rejection of this new configuration with respect to the uncontrolled system can be written as:

$$
S(j \omega)=\frac{P_{c l}(j \omega)}{P(j \omega)}=\frac{1}{1+P C}
$$

whereas the sensitivity, $S(j \omega)$, can be graphically interpreted as the inverse of the vector $P C(j \omega)$ to the point minus one in the Nyquist plot (Fig. 4). Eq.(5) shows that an open-loop transfer within the unitcircle around -1 results in performance degradation with respect to the uncontrolled system, known as spill-over $(|S(j \omega)|>1)$ [19]. An open-loop transfer outside this circle results in an increasing performance for the corresponding frequency $(|S(j \omega)|<1)$.

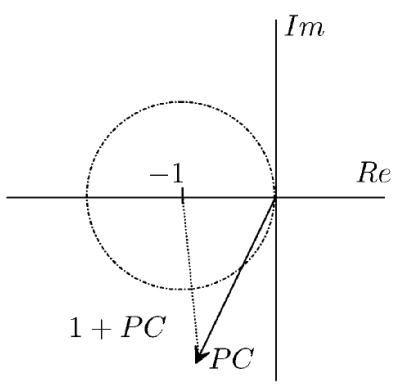

Figure 4: Graphical interpretation of $S(j \omega)$

Note that the local-performance specified by Eq. (5) does not imply global performance. It is shown in [2] that high feedback gains, i.e. a low sensitivity, lead to much disturbance rejection in the loop however also makes that the controlled DoF becomes fixed. As a result, situation 5a behaves like 5b for high feedback gains. This makes the system sensitive for disturbances acting on other DoFs than the controlled ones. So in order to reduce vibration levels of the whole plate, the objective is not to minimize local displacement but maximize local energy dissipation from dominant modes and consequently introducing damping as mentioned in section 3 .

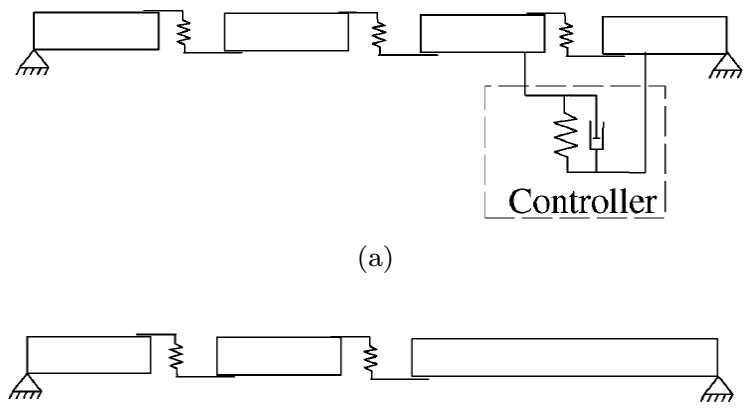

(b)

Figure 5: High feedback gains in (a) results in undamped behaviour of (b).

\subsubsection{Stability}

Despite the lack of open-loop tuning to tune for global performance, the open-loop is still very valuable for stability analysis via Nyquist stability criterion since it can be directly based on measurement data and therefore does not suffer from modelling errors like root-locus inevitably does. Measurements of the open-loop FRF include all effects like time-delay in the control-system and amplifier dynamics which can tread stability, especially at higher frequencies.

Using positive feedback, the stiffness of the mechanical system line in the low frequent region appears at $-180^{\circ}$ in the Nyquist plot. This makes that low-frequent behaviour becomes the limiting factor for stability, resulting in a non-dynamic stability criterium according to the Nyquist stability criterium [13]. Using the Nyquist-plot, it can be derived that a necessary condition for stability is:

$$
|P C(\omega=0)|<1
$$

Eq.(6) shows that the static controller gain is restricted by the stiffness of the plant.

This static-stability criterium is closely related to the well known fact that PPF reduces the stiffness of the closed-loop plant at low frequencies. Because the open-loop starts at $180^{\circ}$, it can directly be derived from Eq.(5) that spill-over effects occur in this frequency region.

\subsubsection{Limits of performance}

Besides stability, open-loop analysis shows that the achievable local performance is limited by the sensitivity function. Assuming that there are at least two more poles than zeros, the bode-sensitivity integral holds [13, 19].

$$
\int_{0}^{\infty} \log |S(j \omega)| d \omega=0
$$

It follows from Eq.(7) that the surface under a controlled FRF is invariant and therefore shows a funda- 
mental bound on the controller performance. Combining Eq.(5) and (7) gives:

$$
\int_{0}^{\infty} \log |S| d \omega=\int_{0}^{\infty} \log \left|P_{c l}\right| d \omega-\int_{0}^{\infty} \log |P| d \omega=0
$$

The maximum value of the transfer function $P_{c l}(j \omega)$ is often determining for performance. As a consequence, a constant FRF would be optimal. If it is supposed that the transfer function can only be manipulated till a certain frequency $f_{u}$, this constant of optimal performance can be derived after manipulating Eq.(8)

$$
\left|P_{c l}\right|=10^{\frac{f_{0}^{f_{u}} \log |P| d \omega}{f_{u}}}
$$

This line is depicted in Fig. 2. Further reduction can not be obtained by feedback. However for badly damped systems, this fundamental upper-bound for performance will not be a limiting factor.

Note that for noise radiation problems an Aweighted constant would be more appropriate. This curve can be derived by multiplying Eq.(8) with the inverse frequency response of an A-weighting.

\subsubsection{Open-loop for damping}

Controllers proposed for active damping such as VF and $\mathrm{PPF}$ add respectively $+90^{\circ}$ or $-270^{\circ}$ phase in the open-loop. This supposes that this phase-shift in the open-loop is responsible for optimal damping. This can be understood since the measured velocity and the applied control-force are $180^{\circ}$ out of phase implying energy dissipation. However in closed-loop, the dynamic controller properties change due to feedback by the plant such that expressions about optimal phase for energy dissipation are harder to make using information of the open-loop.

Instead of open-loop, root-locus tuning is used to maximize energy dissipation in particular modes.

\subsection{Root-locus}

A measure for the energy dissipation from a certain mode can be found in the damping of the closed-loop poles represented by the angle $\beta$ with the imaginary axis of the complex plane as plotted in Fig. 6 and 7 . The damping of these modes is maximized in the control loop using piezo pair $1\left(\frac{Y}{U}\right)$, which results in an increasing performance at other places $\left(\frac{Z}{D}\right)$ as mentioned in section 3. As a consequence, $\beta$ can be used as tuning criterium to place the closed loop poles. These closed-loop poles are calculated using the obtained model for $\frac{Y}{U}$ as derived in section 3 .

Having positive feedback, damped closed-loop poles attract poles of undamped modes which are in the same frequency region. Therefore placement of damped closed-loop poles, coming from the controller, can be used to attract the undamped poles further into the left half-plane. However during the placement of the controller poles, i.e. choosing the controller parameters, several trade-offs appear.

The tuning of the controller, represented by the parameters $\omega_{c_{n}}, \xi_{c_{n}}$ and $G_{n}$, is done by hand in an iterative way based on the root-locus plot and the FRF of the open-loop. The influence of the parameters on the root-locus plot and it's relations to the expected damping in several modes will be shortly discussed and illustrated.

The influence of the gain variable is intrinsically depicted in the root-loci plot. Since closed-loop poles start at the undamped open-loop poles and end in the undamped zeros $[13,2]$, there exist an optimum as already discussed in subsection 5.1.1. However this optimal gain can differ per mode such that an average has to be found over the modes weighted with their dominance while still remaining stability according to the open-loop stability criterion (Eq.(6)). The relative gain between the PPF-modes, which determines the placement of the controller zero's, can also be used to shape the root-loci whereas the rule of thumb given in subsection 5.3 can be useful.

The influence of the parameter $\omega_{c_{n}}$ is rather straightforward since it makes the damping work either in a lower or higher frequency region. Note that the placement of the closed-loop poles originated from the controller are determining for damping such that, according to Fig. 6 and 7 , the controller pole is in general placed higher frequent than the region where much damping is achieved.

With respect to damping, there appears to be a trade-off between placing a closed-loop pole close to an undamped pole resulting in maximum attraction of that pole [9], as depicted by the dotted root-loci in Fig. 7, or choosing to use more damped poles such that the attraction of the damped closed-loop pole is spread over more undamped closed-loop poles which is illustrated by the continuous root-loci in Fig. 7 . Note that further increase of damping results in loss of effectiveness meaning that undamped poles are less attracted into the left half-plane. Due to high amount of damping, less gain is generated by the resonance of the controller resulting in a demand for higher static gain which can tread stability.

Fig. 6 and 7 shows the root-locus of the tuned system using one PPF DoF and two PPF DoFs, respectively. The controller parameters are tuned to suppress the dominant modes of $\frac{Z}{D}$ which, by symmetry, also equal the dominant modes of $\frac{Y}{U}$. By placing damped controller poles and zero's, damped closedloop poles are generated that attract poles of modes which were initially undamped.

Parameters which results in a broad-band reduction of the dominant resonances of piezo pair 6 , are given in Table 2. Fig. 6 and 7 show that the maximum achievable modal damping of both controllers is comparable. It is expected that if the dominant reso- 
nance peaks are more grouped in several frequency regions, a summation of several controller modes could be more advantageous compared to the use of one controller mode. Another option to achieve broadbanded reduction, is to tune each piezo for damping in another frequency region using single DoF PPF controllers with multiple loops [20].

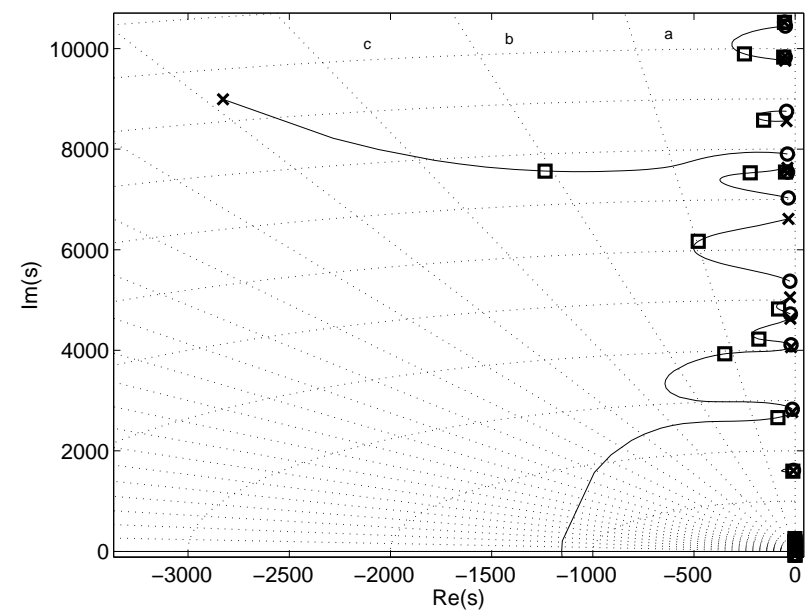

Figure 6: Root-locus of piezo pair 1 using one PPF DoF

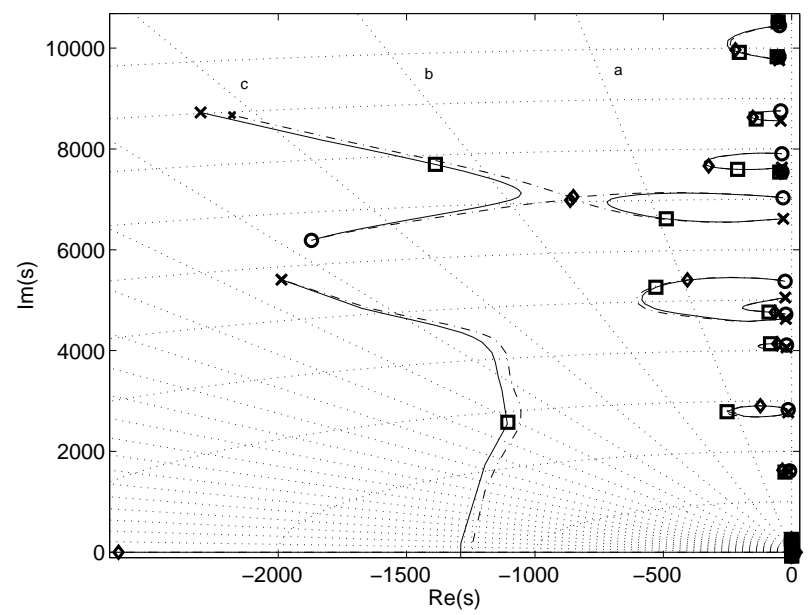

Figure 7: Root-locus of piezo pair 1 using two PPF DoFs, (-) Controller focussed on broad-band reduction, (-.) Controller focussed on one particular mode (closed-loop poles indicated by $\square$ and $\diamond$ respectively)

Note that the maximum achievable damping depends on the distance between poles and zeros in the root-locus $[2,9]$, i.e. observability and controllability of particular modes. High modal density and high residual terms leads to close poles and zeros spacing which limits the achievable damping for distributed parameter systems.

Trade-offs were needed to find the optimal tuning for several modes. One could wonder if the usage of
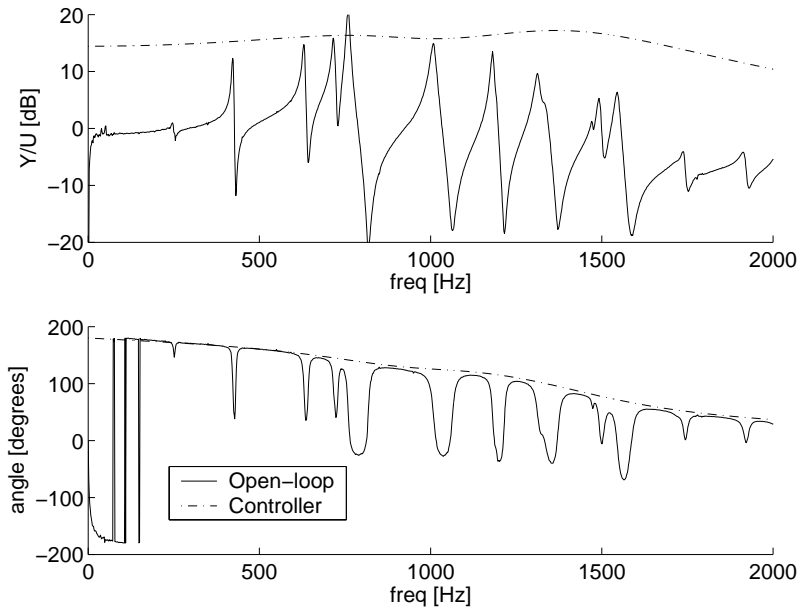

Figure 8: Bode-plot of measured open-loop using piezo pair 1 with 2 PPF DoFs

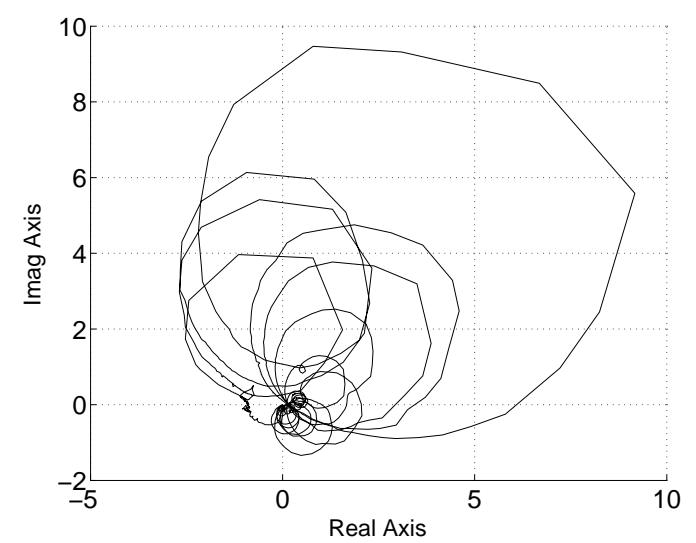

Figure 9: Nyquist of measured open-loop for piezo pair 1 using 2 PPF DoFs

more PPF modes would be beneficial since the additional freedom can better meet these trade-offs. However this has several drawbacks. Due to the summation of PPF modes, less gain is possible per mode due to the low-frequent stability criterion. This makes that the PPF modes of the controller have to be less damped in order to achieve enough gain at the frequency of interest. This makes the performance less robust to changes in dynamics behaviour of the plant. On the other hand, the resulting high order controllers lead to practical problems as already mentioned in the introduction.

\subsection{Best of both worlds}

Optimal gain is reached if the open-loop crosses the $0 \mathrm{~dB}$ in the middle of the logarithmic frequency scale between anti-resonance- and resonance [1]. This additional condition can be seen as a rule of thumb to find the optimal gain tuning of the controller. This 
correspond with Fig. 8, which shows the bode plot of the open-loop of the tuned system using a controller with two degrees of freedom.

Fig. 9 depicts the corresponding Nyquist plots of the open-loop for positive frequencies using a controller with 2 DoFs as tuned according to Fig. 7. Note that the resonances of the stable open-loop FRF lead to phase loss and therefor to resonance circles that run clockwise in the Nyquist plot. Fig. 9 supposes that damping is optimal if the resonances lie in the first quarter of the Nyquist-plot, which can be explained from the fact that the controller still reduces local stiffness, leading to higher local deformations, while starting the damping action by shifting the phase of the controller. This again shows a trade-off between fulfilling this phase condition for a broad frequency band implying much damping which results in loss of effectiveness and local fulfilment resulting in adding damping in one particular mode.

As a fine tuning, the controller parameters can be slightly varied while measuring the amount of damping in loop 6 using the FRF of $\frac{Z}{D}$ according to Fig. 1(b). Based on the open-loop, the root-locus plot and fine-tuning on the system, the following controller parameters are chosen for experimental evaluation:

Table 2: Controller parameters

\begin{tabular}{|c|ccc|}
\hline & $G_{n}$ & $\omega_{n}$ & $\xi_{n}$ \\
\hline 2 DoF's & & & \\
\hline mode 1 & $5.8 \cdot 10^{7}[-]$ & $915[\mathrm{~Hz}]$ & $0.35[-]$ \\
mode 2 & $2.6 \cdot 10^{8}[-]$ & $1435[\mathrm{~Hz}]$ & $0.25[-]$ \\
\hline 1 DoF & & & \\
\hline mode 1 & $5.6 \cdot 10^{8}[-]$ & $1499[\mathrm{~Hz}]$ & $0.3[-]$ \\
\hline
\end{tabular}

\section{$6 \quad$ Experimental results}

The following experimental equipment is used: Dspace Alfa Combo Dual Processor Board used for controller implementation at $20 \mathrm{kHz}$, Siglab 5021 used for data acquisition and Polytec scanning laser vibrometer for measuring Operating Deflection Shapes (ODS) analysis. The input voltage to the actuators is amplified whereas the output voltage of the sensing piezo's is directly fed to the devices.

A $4^{\text {th }}$ order PPF controller using 2 DoFs, tuned according to Fig. 7 and Table 2, is implemented. The control loop is closed around piezo pair 1 whereas the performance is evaluated by measuring the FRF at piezo actuator/sensor pair 6 . Fig. 10 shows the FRF of piezo pair 6 for the controlled and uncontrolled case.

Note that the low-frequent reduction of stiffness is not observed at piezo pair 6 which shows that reduction of stiffness is only a local effect.
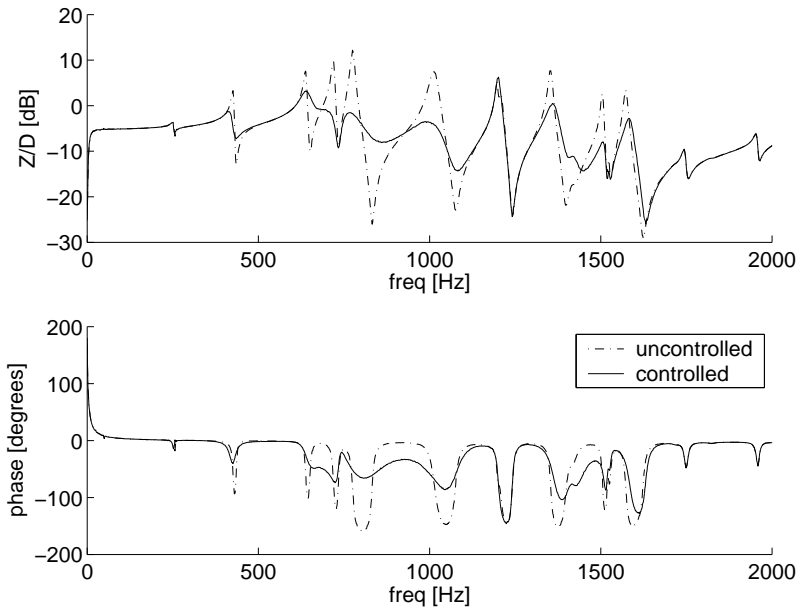

Figure 10: Measured Frequency Response Function $\frac{Z(j \omega)}{D(j \omega)}$

Tabel 3 shows that reduction in vibration level of modal peaks is achieved for a broad frequency band, up to $13.8 \mathrm{~dB}$ for the $(2,2)$ mode. Remark that not all frequencies are well damped. Further investigations using scanning laser vibrometer measurements show that the mode at $1190 \mathrm{~Hz}$ is not a structural mode but an air mode inside the box which is apparently harder to damp.

Nevertheless, Tabel 3 shows that the maximum peak value of the transfer-function is reduced by 6.2 $\mathrm{dB}$. If only structural modes are taken into account, a reduction of the maximum peak value of $9.1 \mathrm{~dB}$ is achieved.

Resonances at high frequencies puts high demands on digital equipment with respect to sampling and computational delay. The resulting time-delay causes phase lag which rotates the resonances in the Nyquist open-loop plot into the circle around -1 leading to spill-over effects according section 5.1. These high frequent spill-over effects appear in the $4 \mathrm{kHz}$ region where the controller does not have enough roll-off to cancel delay-effects (Fig. 11).

The relative low complexity of the controller makes it possible to implement these controllers analogue $[21,22]$. This overcomes the problem of time-delay and on the other hand opens possibilities to extent damping to much higher frequencies than could be achieved by means of digitally implemented controllers. Experiments have been done with Universal State Variable filters and Switched Capacitor Filters [22] leading to comparable results. Fig. 11 shows that spill-over effects in the $4 \mathrm{kHz}$ range are vanished using an analogue implementation.

To improve the flexibility of the analogue implementation, digitally controlled potentiometers could be used to vary the parameters of the analogue filter. This combines the flexibility of digital implementation and the advantages of analogue filters with respect to 


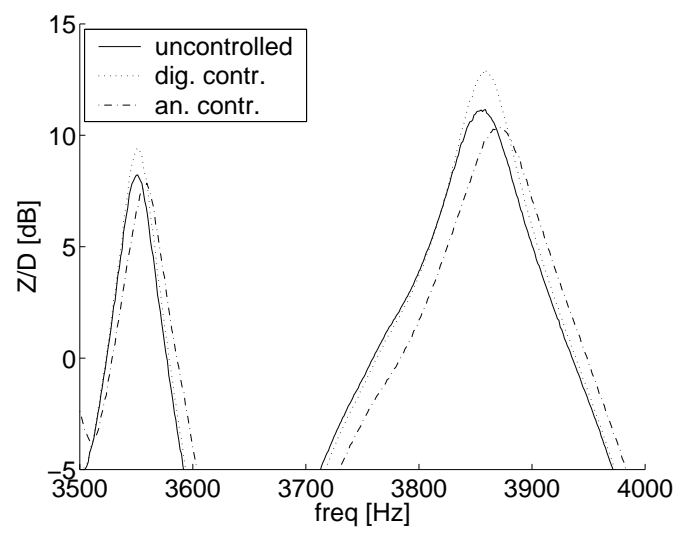

Figure 11: Measured high frequent spill-over effects

time-delay.

An ODS scan is made in order to quantify the reduction of vibration levels over the entire plate. Fig. $12 \mathrm{a}$ and $12 \mathrm{~b}$ shows a global reduction of $12 \mathrm{~dB}$ in the ODS at $1 \mathrm{kHz}$. This proves that global vibration levels can be reduced by locally added damping. Fig. 13 shows the appearance of complex modes for the controlled system opposed to real modes for the uncontrolled structure. This confirms that a significant amount of damping is added by the controller.

As a confirmation of global broad-band vibration suppression, an acoustic reduction in the order of 5 $\mathrm{dB}$ is measured during excitation with random noise in the frequency range till $1 \mathrm{kHz}[23]$.

Table 3: Modal reductions

\begin{tabular}{|c|c|c|c|}
\hline mode $(l, w)$ & freq $[\mathrm{Hz}]$ & $\begin{array}{c}\text { max } \\
\text { uncontr. }[\mathrm{dB}]\end{array}$ & $\begin{array}{c}\text { max } \\
\text { contr. }[\mathrm{dB}]\end{array}$ \\
\hline$(1,1)$ & 228 & -3.4 & -3.7 \\
$(2,1)$ & 424 & 3.3 & -1.3 \\
$(1,2)$ & 634 & 7.6 & 3.2 \\
$(3,1)$ & 720 & 9.6 & -1.0 \\
$(2,2)$ & 763 & 12.3 & -1.5 \\
$(3,2)$ & 1014 & 7.5 & -3.5 \\
air & 1189 & 5.5 & 6.2 \\
$(2,3)$ & 1338 & 7.6 & 0.5 \\
$(4,2)$ & 1504 & 3.0 & -8.0 \\
$(3,3)$ & 1556 & 4.0 & -3.0 \\
$(5,1)$ & 1754 & -9.6 & -9.5 \\
$(1,4)$ & 1938 & -6.0 & -6.5 \\
\hline
\end{tabular}

\section{Conclusion}

It is shown that broad-band vibration suppression can be obtained for mechanical distributed systems using low-authority controllers. Modelling shows that global performance can be reached by locally adding damping into the modal components. Both open-loop

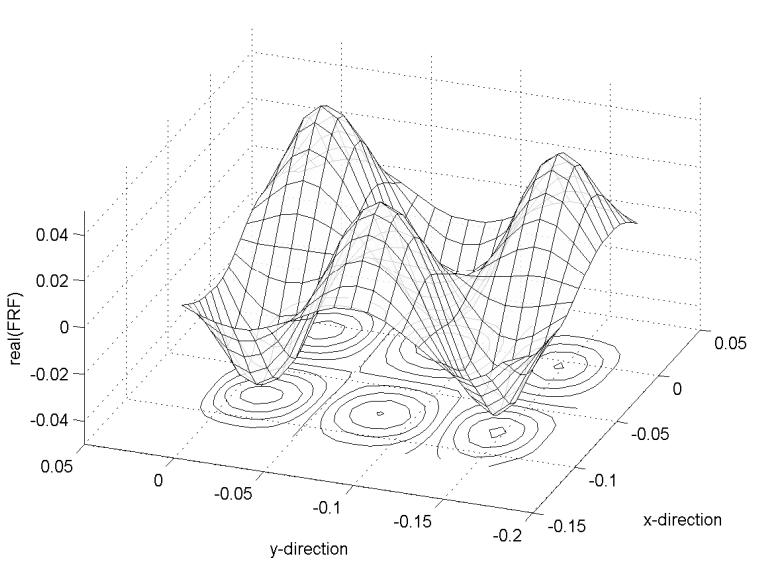

(a)

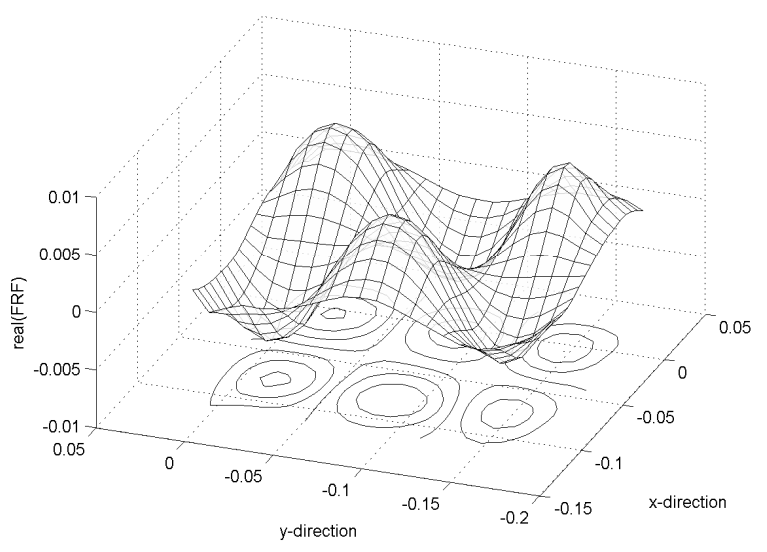

(b)

Figure 12: ODS measured velocities at $1 \mathrm{kHz}$ of uncontrolled (a) and controlled (b) system

and root-locus tuning were used to assure stability and maximize performance.

Open-loop appeared to be valuable to guarantee stability, leading to a necessary non-dynamic stability criterium and prediction of spill-over effects especially in the low-frequent region. The bode-sensitivity integral shows that the performance of feed-back is constrained to a fundamental bound.

Maximizing modal damping is translated into a root-locus tuning criterium. A trade-off appears between putting less damping into the closed-loop poles (coming from the controller) resulting in attraction of one particular mode into left half-plane or adding more damping such that attraction is spread over more modes, thereby losing a part of the attraction.

A $4^{\text {th }}$ order PPF controller is tuned and experimentally evaluated for broad-band vibration suppression by closing a control-loop over one collocated piezo pair. Frf's and ODS measurements show that broadband vibration suppression is achieved. Vibration reductions up to $13.8 \mathrm{~dB}$ are achieved for particular modes whereas the maximum value of the FRF is re- 


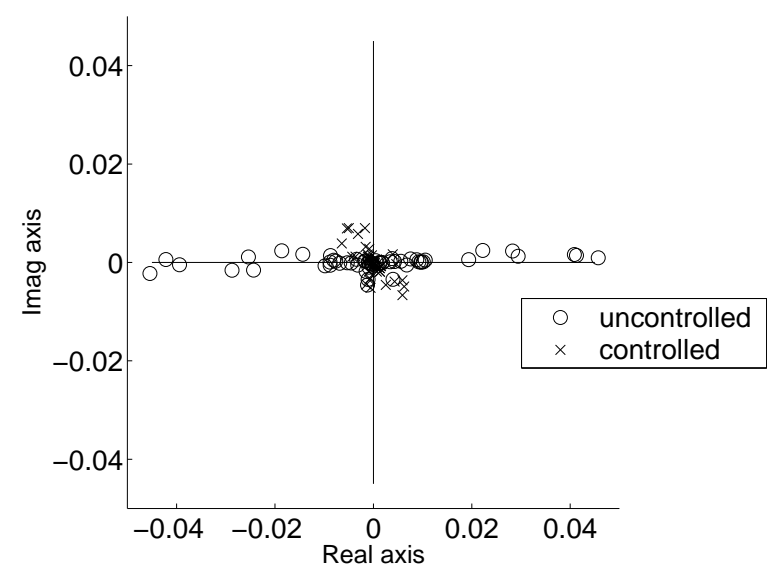

Figure 13: Measured Frequency Response Functions of velocities at ODS grid-points at $1 \mathrm{kHz}$ of uncontrolled and controlled system

duced by $9 \mathrm{~dB}$ for structural modes.

During experiments, the limitation of a digital implementation becomes visible in terms of spill-over effects in the high frequent region. An analogue implementation of the low order PPF controllers successfully reduced these high frequent spill-over effects and opens new possibilities to extend the frequency range of control.

\section{Acknowledgments}

The authors like to thank the section of Applied Mechanics of the University of Twente for the design of the measurement setup and the development of a coupled piezo/mechanical FEM modelling approach.

\section{References}

[1] M. P. Koster, Design Principles for Accurate Motion and Positioning (in Dutch). Enschede: Twente University Press.

[2] A. Preumont, Vibration Control of Active Structures. Université Libré de Bruxelles: Kluwer Academic Publishers, 2002.

[3] J. N. Aubrun, "Theory of the control of structures by low-authority controllers." AIAA J. of Guidance, vol. 3, no. (5), 1980.

[4] M. J. Balas, "Direct velocity feedback control of large space structures." AIAA J. of Guidance, vol. 2, no. 3, 1979 .

[5] J. L. Fanson and T. K. Caughey, "Positive position feedback control for large space structures." AIAA Journal, vol. 28, no. 4, 1990.
[6] D. Halim and S. Moheimani, "Experimental implementation of spatial $\mathcal{H}_{\infty}$ control on a piezoelectric-laminate beam," IEEE/ASME Trans. Mechatron., vol. 7, no. 3, pp. 346-356, September 2002.

[7] J. Holterman, "Vibration control of highprecision machines with active structural elements," Ph.D. dissertation, University of Twente, 2002.

[8] D. Halim and S. Moheimani, "Spatial $\mathcal{H}_{2}$ control of a piezoelectric laminate beam: Experimental implementation," IEEE Trans. Contr. Syst. Technol.

[9] M. A. McEver, "Optimal vibration suppression using on-line pole/zero identication," Master's thesis, Virginia Polytechnic Institute and State University, December 1999.

[10] A. den Hamer, G. Z. Angelis, N. B. Roozen, and R. v.d. Molengraft, "Broad-band active vibration suppression," in $23^{\text {th }}$ Benelux Meeting on Systems and Control, 2004.

[11] R. Visser, "Experimental validation of a boundary element solver for exterior acoustic radiation problems," Proceedings $10^{\text {th }}$ Int. Congress on Sound and Vibration, pp. p1499-1506, 2003.

[12] W. K. Gawronski, Advanced Structural Dynamics and Active Control of Structures. Springer, 1998.

[13] G. Franklin and J. Powell, Feedback Control of Dynamic Systems. Addison-Wesley, 1994.

[14] A. Koevoets, G. Angelis, and N. Roozen, "Modeshape based predictive piezo modelling evaluated on a plate with piezoelectric-array," in Proceedings of $11^{\text {th }}$ Int. Congress on Sound and Vibration, 2004.

[15] S. Gangbing Song, Schmidt and B. Agrawal, "Experimental study of active vibration suppression of flexible structure using modular control patch," Aerospace Conference, vol. Proceedings., IEEE, no. Volume: 1, pp. 189 -201 vol.1, March 1998.

[16] J. Bell and R. Kashani, Eds., Structure-Borne Noise Reduction by Active Damping of a Radiating Panel. Proceedings of the SAE Noise and Vibration Conf., May 15-18 1995.

[17] T. de Kort, "Active silence," Master's thesis, Section of Applied Mechanics and Polymer Engineering, August 2002.

[18] M. Friswell and D. J. Inman, "The relationship between positive position feedback and output feedback controllers," Smart. Mater. Struct., vol. 8, pp. 285-291, 1999. 
Preprint submitted to IEEE/ASME TRANSACTIONS ON MECHATRONICS, 2005, Den Hamer, Angelis \& Roozen, p. 10

[19] J. Hong and D. Bernstein, "Bode integral constraints, collocation, and spillover in active noise and vibration control," IEEE Trans. Contr. Syst. Technol., vol. Volume: 6, no. Issue: 1, pp. $111-$ 120, Jan. 1998.

[20] N. Tanaka and S. D. Snyder, "Cluster control of a distributed-parameter planar structure-middle authority control," J. Acoust. Soc. Am., vol. 112, no. 6, pp. 2798-2807, December 2002.

[21] N. Balabanian, Electric Circuits. McGraw-Hill, 1994.

[22] P. Horowitz and W. Hill, The Art of Electronics, 2nd ed. Cambrige University Press, 1998.

[23] G. van Schothorst, M. Vermeulen, and G. Angelis, "Piëzo-elektrische aandrijving in mechatronisch systeemontwerp (in dutch)," Mikroniek, 2004. 